\title{
The Structure of the Head, Collar and Base-Plate of ' $T$-even' Type Bacteriophages
}

\author{
BY D. E. BRADLEY \\ Department of Zoology, University of Edinburgh, Scotland
}

(Received 22 September 1964)

\begin{abstract}
SUMMARY
A major feature in the classical conception of the morphology of the T-even type coliphage is the shape of the head, believed to be a bipyramidal hexagonal prism. The present paper shows that this shape is not consistent with the appearance in electron micrographs, and an alternative model is proposed. The fine structure of the head is compared with that of polymerized head protein (polyhead) obtained from naturally-occurring lethal mutants. Certain hexagonal and star-shaped objects have been described in the literature as base-plates. Because of inconsistencies in their appearance, they have now been measured and studied. The results given here indicate that the star-shaped objects are disorganized base-plates and the hexagonal objects are collars. By using the rotation method for printing micrographs of collars it is found that they have a hollow subunit at each corner.
\end{abstract}

\section{INTRODUCTION}

Despite the fact that coliphages of the T-even morphological type have been studied more than any other kind, several questions about their molecular structure remain to be answered. This is mainly because they are complex bodies with numerous structural components, whose functions are in many cases uncertain. This paper seeks to add further details to the picture which has been built up in the past.

Investigations hitherto have shown that the T-even type phage consists of a head, believed to be in the form of a bipyramidal hexagonal prism (Williams \& Fraser, 1953) and a complicated contractile tail, which has been the subject of the most intensive study. The tip of the tail consists of a hexagonal base-plate with a pin attached to each corner. A thin hollow tube or core is firmly attached to the base plate and runs up the centre of the tail to the head of the phage where it is fixed to the apex of one of the pyramids of the bipyramidal hexagonal prism. A sheath which forms the contractile part of the tail surrounds the core and extends almost to the head (Brenner et al. 1959). In the normal or extended state, i.e. before nucleic acid injection, it consists of some 144 morphological subunits related by screw symmetry and arranged in a series of 24 annuli of sixfold radial symmetry (Bradley, 1963). It is believed that the sheath contraction which accompanies nucleic acid injection is brought about by doubling the number of subunits per annulus (from six to twelve), a hypothesis which is supported by observations with the electron microscope. Between the top of the sheath and the head there is a disc-like collar (Anderson, 1960; Bradley, 1963) which almost certainty has a sixfold radial symmetry. Several fibres, which correspond to the tail fibres seen by Brenner et al. 
(1959), are attached to both collar and base-plate, and form a network round the sheath. These fibres are closely associated with the adsorption process; under conditions when the phage can adsorb and infect, the network breaks at the collar, and the fibres attached only at the base-plate can wave about at random in the medium. As soon as they touch the host bacterium the base-plate is brought into intimate contact with the cell wall. If the phage is placed in a medium in which adsorption cannot take place (e.g. in the absence of suitable cofactors such as tryptophan for coliphage $T$ 4) the fibres are found as the network round the sheath (Professor E. Kellenberger and colleagues, personal communication). The phage head contains double-stranded deoxyribonucleic acid (DNA) which can be unravelled and seen in the electron microscope as a single filament $49 \mu$ long (Kleinschmidt, Lang, Jacherts \& Zahn, 1962). One free end of this fibre extends down the inside of the core to the base-plate.

The structures of a few of these features have yet to be fully described or clarified. The most important is the phage head; the number, size and arrangement of the morphological subunits or capsomeres are still unknown. Also, there are certain features in electron micrographs of the head which strongly suggest that the classical model for its geometrical form is incorrect or at least that the head is pleomorphic. In the tail, the only features which are not clearly described are the collar and the detailed structure of the base-plate. These points are discussed here on the basis of electron micrographs.

Preparations of single phage components for electron microscopy may be obtained either by chemical and physical separation (Brenner et al. 1959; Anderson \& Stephens, 1964) or from lysates of lethal mutations of the phage. Artificiallyinduced lethal mutants of coliphage $T 4$ were studied by Epstein et al. (1964), Favre et al. (1964) and Eiserling \& Boy de la Tour, 1964). They showed amongst other things that in such cases only one phage component or component protein may be produced in the host bacterium. Some mutants caused the host cell to produce tail cores with base-plates attached, while others induced the formation of sheath or head protein. The latter were called polysheath and polyhead, respectively. Since both are chemically and serologically identical with the true phage components, one might expect a structural relationship to exist. These molecular assemblies are easy to identify in electron micrographs of lysates or sections of infected bacteria and are described here in association with new isolates of T-even phages, indicating that such mutants can occur naturally.

\section{METHODS}

Sources of coliphages. Coliphage BP/4 was isolated from the River Ayr at Ayr; phage PVL was obtained from a manure heap near Biggar, Lanarkshire; and phage WB/5 from a canal in Vienna. Coliphage T 4 was supplied by Dr and Mrs K. G. Lark (Kansas State University).

Isolation, growth and purification of bacteriophages. The methods used were the same as those described previously, with Escherichia coli strain c 2 as a host organism (Bradley, 1963). Suspensions for electron microscopy were made by extracting confluently lysed plates with neutral $0 \cdot 1 \mathrm{M}$-ammonium acetate. Purification was by several cycles of centrifugation. 
Treatment of coliphage $\mathbf{T} 4$ with antiserum. Anti-T 4 serum was kindly supplied by Dr J. G. Howard (Edinburgh University). A phage suspension in broth was decreased to about $25 \%$ of its original activity by adding a suitable dilution of antiserum and incubating at $37^{\circ}$ for $15 \mathrm{~min}$. The phage particles were then centrifuged at $15,000 \mathrm{~g}$ for $1 \mathrm{hr}$ and resuspended in $0 \cdot 1 \mathrm{M}$-ammonium acetate (neutral) for electron microscopy.

Electron microscope specimen preparation. The negative contrast method was used; most of the embedding mixtures have been mentioned elsewhere (Bradley, 1962). Solutions of uranyl salts containing ethylenediaminetetra-acetic acid (EDTA) were most suitable for the preservation of the shape of the phage head. Uranyl formate (Finch, 1964) required a special procedure for the preparation of a $2 \%$ solution, the reason being that this compound is not easily obtainable from the major chemical manufacturers. It is best prepared in the laboratory as follows. Uranyl nitrate (cryst.; $2.79 \mathrm{~g}$.) is dissolved in about $30 \mathrm{ml}$. water and ammonium hydroxide added to excess. The resulting canary yellow ppt. of uranyl hydroxide is centrifuged at $2000 \mathrm{~g}$ for $2 \mathrm{~min}$. and the colourless supernatant fluid discarded. The ppt. is washed free from ammonia by centrifugation and finally resuspended in about $30 \mathrm{ml}$. water. The addition of $0.5 \mathrm{ml}$. of $90 \%$ formic acid will just dissolve the uranyl hydroxide, providing a solution of uranyl formate, which on making up to $100 \mathrm{ml}$. will contain $2 \%(\mathrm{w} / \mathrm{v})$. This solution can be applied to grids in the normal way, but it is not stable, a ppt. forming within a few days. It is therefore best to make a fresh solution when required.

Printing electron micrographs. To obtain greater detail from some of the electron micrographs obtained, the rotation method was used for printing them (Markham, Frey \& Hills, 1963). Suitable areas were first selected by rotating normal prints at several hundred rev./min. under stroboscopic illumination. The negatives were then placed in the enlarger, the selected areas centred over a gramophone turntable, and the printing paper placed on the turntable. Since the specimens concerned obviously possessed a sixfold rotational symmetry, prints were made by using six exposures, the turntable being rotated one sixth of a revolution between each. Particular care is required in the interpretation of such prints.

\section{RESULTS}

\section{The shape of the normal phage head}

Electron micrographs of the head showed features which were inconsistent with the classical model of a bipyramidal hexagonal prism. These consisted of rhomboids and other shapes on the faces of the main body of the head, supposedly a right hexagonal prism; typical examples are shown in Pl. 1, fig. 1. More or less similar shapes can be found in some published micrographs (e.g. Brenner et al. 1959, Pl. IV; Cummings \& Kozloff, 1962; Anderson \& Stephens, 1964, fig. 4). In Pl. 1, fig. 2, the supposed prism has a triangular face on it. On the other hand, profiles of phage particles where the support film has folded (Pl. 1, fig. 3) would appear to be those of the classical figure; therefore any proposed new shape would have to be close to a bipyramidal hexagonal prism and difficult to distinguish from it. Such a figure can be deduced from electron micrographs and can be obtained by rotating one pyramid by $30^{\circ}$ with respect to the other and replacing the prism by a series of triangles as 
shown in the model in Pl. 1, fig. 4. The slight departure from the vertical of the outer edges of the main body of Pl. 1, fig. 4 (left) is due to tilt in the copying camera; this would not show with the virtually parallel illumination of the electron microscope.

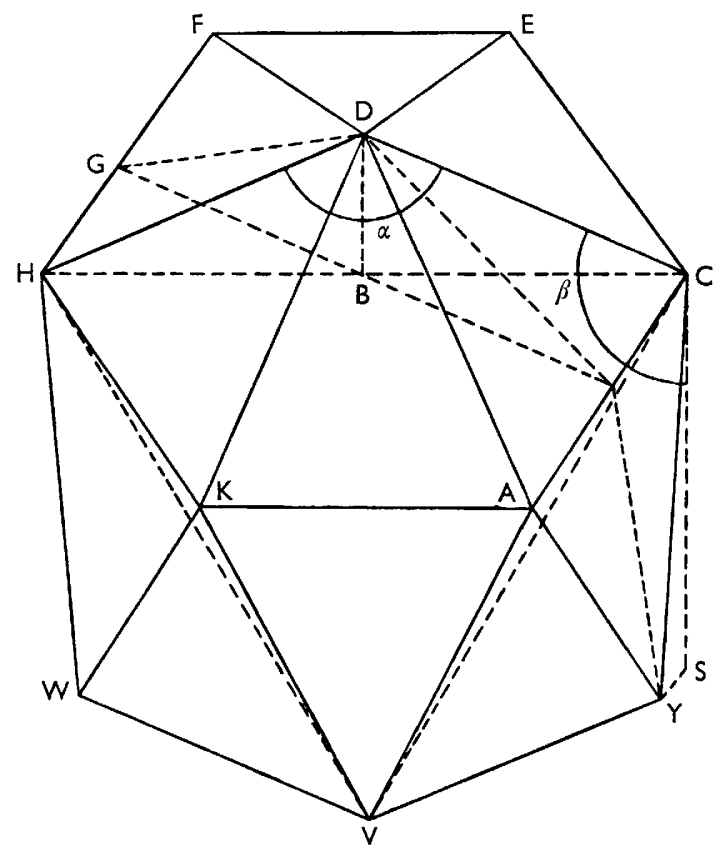

$a$

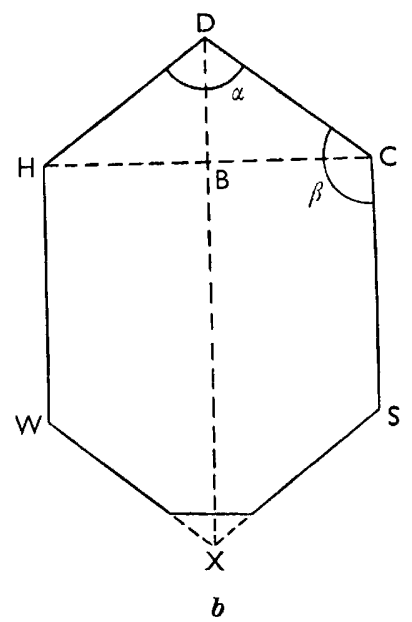

Fig. 1. (a) Diagram of part of proposed figure for T2 type phage heads; CS is perpendicular to the plane of the hexagon EFHKAC; it represents the projection of an edge (e.g. CY) when the whole figure is rotated in either direction by $30^{\circ}$ to correspond to $\mathrm{Pl} .1$, fig. 4 (left). (b) Diagram of outline of phage head as seen in electron micrographs.

In the heads in Pl. 1, figs. 5, 6 several faces are visible; by no stretch of the imagination can they be attributed to a hexagonal prism, but the superimposed wire models of the proposed figure are much closer. Some of the edges in the models have been erased and replaced by broken lines to accentuate the sides corresponding to those visible in the micrographs. One frequently finds the top of the phage head wider than the bottom, or vice versa (Pl. 1, fig. 7; see also Brenner et al. 1959, Pl. III), and again the wire model shows how this can come about, though the pyramids of the model are more acute than those of the phage, probably due to distortion. The reliability and implications of these observations are discussed below.

\section{The dimensions of the normal phage head}

To prepare a model of a normal phage head and to consider its structure the various dimensions must be ascertained. This has been achieved by taking measurements from micrographs of phages in various negative-staining media and averaging the results. The measurements are given in full in Table 1 , the various sides and angles referring to Fig. 1, which is drawn from the model described above. 
To test further the validity of the model, the acute angles which are indicated by arrows in Pl. 1, fig. 1, were measured on a number of micrographs; these corresponded to the angles AVY or KWV in Fig. 1. The results are given in Table 2, and their significance is discussed below. It was found that the best negative-staining media for the three-dimensional preservation of large objects were uranyl acetate with EDTA and a mixture of sodium molybdate and ammonium carbonate (Bradley, 1962). On the whole, T 2 type heads tend to collapse in phosphotungstate (PTA) so that the majority of heads whose measurements are given in Table 1 and 2 were embedded in the uranyl or molybdate media.

Table 1. Dimensions of T2 type phage heads (see Fig. 1)

\begin{tabular}{|c|c|c|c|c|c|c|c|c|}
\hline $\begin{array}{c}\text { Phage } \\
\text { type }\end{array}$ & Negative stain & a & $3^{\prime}$ & DC & $\mathbf{D X}$ & CS & DB & $\mathbf{H C}$ \\
\hline T4 & Na molybdate & 117 & 121 & 4,10 & 1000 & 480 & 260 & 710 \\
\hline $\mathbf{T} 4$ & Na molybdate & 107 & 126 & 430 & 1100 & 570 & 263 & 710 \\
\hline$\phi 66 t-$ & PTA & 96 & 132 & 560 & 1350 & 600 & 375 & 825 \\
\hline$\alpha 1$ & $\mathbf{P}^{\prime} \mathbf{T A}$ & 108 & 131 & 530 & 1350 & 660 & 345 & 810 \\
\hline T2 & Uranyl nitrate & 107 & 125 & 490 & 1150 & 550 & 300 & 725 \\
\hline T2 & Uranyl nitrate & 109 & 125 & 460 & 1100 & $\mathbf{5 5 0}$ & 275 & 725 \\
\hline T2 & Uranyl nitrate + EDTA & 103 & 128 & $\mathbf{5 3 0}$ & 1250 & 580 & 335 & 860 \\
\hline T2 & Uranyl nitrate + EDTA & 117 & 121 & 490 & 1200 & 670 & 265 & 800 \\
\hline T2 & Uranyl nitrate + EDTA & 110 & 124 & 520 & 1300 & 710 & 295 & 815 \\
\hline $\mathbf{T 2}$ & Uranyl nitrate + EDTA & 115 & 122 & 510 & 1200 & 650 & 275 & 850 \\
\hline T2 & Uranyl acetate & 96 & 131 & $\mathbf{5 2 0}$ & 1450 & 700 & 375 & 850 \\
\hline T2 & Uranyl acetate & 118 & 122 & 500 & 1300 & 780 & 260 & 800 \\
\hline T2 & Uranyl acetate + EDTA & 99 & 132 & 600 & 1450 & 650 & 400 & 880 \\
\hline T2 & Uranyl acetate + EDTA & 102 & 129 & $\mathbf{5 5 0}$ & 1400 & 700 & 350 & 900 \\
\hline T2 & Uranyl nitrate shadowed & 105 & 128 & 500 & 1250 & 630 & 310 & 825 \\
\hline \multirow[t]{2}{*}{ T2 } & Uranyl nitrate shadowed & 106 & 129 & 540 & 1350 & 600 & 375 & 850 \\
\hline & Averages & $107^{\circ}$ & $127^{\circ}$ & $510 \AA$ & $1260 \AA$ & $630 \AA$ & 315 A & $810 \AA$ \\
\hline
\end{tabular}

Note: $\alpha$ is average of angles at $D$ and $X ; \beta$ is average of angles at $H, W, C$ and $S ; D C$ is average of $\mathrm{DC}, \mathrm{DH}, \mathrm{WX}$ and $\mathrm{XS} ; \mathrm{CS}$ is average of $\mathrm{CS}$ and $\mathrm{WH} ; \mathrm{DB}$ is calculated from $\mathrm{DB}=\frac{1}{2}(\mathrm{DX}-\mathrm{CS})$; $\mathrm{HC}$ is average of $\mathrm{HC}$ and WS.

Table 2. Values of acute angle indicated in Pl. 1, fig. 1

\begin{tabular}{|c|c|c|c|}
\hline $\begin{array}{l}\text { Phage } \\
\text { type }\end{array}$ & Negative stain & Number and origin of micrographs & Angle $^{\circ}$ \\
\hline $\mathbf{T 2}$ & Uranyl acetate + EDTA & 7 measurements, Bradley, unpublished & $68 \cdot 5 \pm 4 \cdot 5^{*}$ \\
\hline T4 & $\begin{array}{l}\text { Sodium molybdate }+ \\
\text { ammonium carbonate }\end{array}$ & 7 measurements, Bradley, unpublished & $69 \pm 4 *$ \\
\hline T2 & PTA & $\begin{array}{l}1 \text { measurement, Brenner et al. (1959) } \\
\text { Pl. IV }\end{array}$ & 78 \\
\hline T2 & PTA & $\begin{array}{l}1 \text { measurement, Fernandez-Moran } \\
\text { (1962), fig. } 3\end{array}$ & 70 \\
\hline T2 & Potassium silicotungstate & $\begin{array}{l}1 \text { measurement, Anderson \& Stephens } \\
\text { (1964), fig. 1 }\end{array}$ & 78 \\
\hline $\mathbf{T 2}$ & PTA & $\begin{array}{l}1 \text { measurement, Bradley, unpublished } \\
\text { Average of PTA stained particles }\end{array}$ & $\begin{array}{l}74 \\
75\end{array}$ \\
\hline
\end{tabular}




\section{The structure of normal phage heads}

No properly organized capsomere structure can be seen in micrographs of either full or empty phage heads, regardless of the negative-staining medium used. This is in direct contrast to the appearance of polyhead which is described below. In an attempt to reveal some form of ultrastructure the negative stain uranyl formate which had given so much success with tobacco mosaic virus (Finch, 1964) was used. The results (Pl. 2, fig. 8) showed a very fine periodicity (arrowed). In Pl. 2, fig. 9, the lines ran parallel to the edges of the head, producing a whorled effect. A very close examination revealed circular objects which might be disorganized capsomeres.

Empty heads revealed only the familiar ghost appearance; two are included here embedded in sodium molybdate and ammonium carbonate (Pl. 2, fig. 10) to show a small lump of protein within the head at the point of attachment of the tail core. In these two particles the DNA was lost, without sheath contraction.

Plate 2, fig. 11, was obtained in an attempt to mark the sites of individual capsomeres by adsorbing anti-T 4 antibodies to the phage head. A number of short rods can be seen at the top of the micrograph. Their centre to centre spacing is about $70 \AA$, but the diameter of the visible part of the rod (the white portion) is about $50 \AA$. Such rods were frequently found on other micrographs of partly inactivated phage preparations. Their significance will be discussed later.

\section{The structure of polyhead}

The general appearance of the polyhead at low magnification is shown in $\mathrm{Pl} .2$, fig. 12; an intact phage particle and a short length of polysheath are included in the field for comparison. The polyhead is more or less uniform in width save for a few places where it narrows and becomes penetrated or covered by the PTA negative staining medium. A part of this micrograph is enlarged in Pl. 2, fig. 13; it can be seen that there is a mottling on the polyhead which suggests a subunit structure; this is not present on the intact phage head. In cases where the polyhead is well preserved the, arrangement of these subunits can be seen (Pl. 3, fig. 14). It appears that they are packed in a regular hexagonal array, and this can be confirmed by using the rotation method for printing the micrograph; the area marked in Pl. 3, fig. 14, has been treated in this way and is shown in Pl. 3, fig. 15. Here the subunits are well defined and show a remarkably regular packing over a large area. Plate 3 , fig. 16 strongly suggests that they have a regular hexagonal outline (arrows), a shape which is essential to produce the observed packing. Their appearance also indicates that the PTA penetrated them in a direction perpendicular to the plane of the polyhead surface, suggesting a tubular form. A glance at the right-hand edge of the polyhead in Pl. 3, fig. 14, where the subunits are more or less in profile, confirms this.

The widths of the cylindrical portions of pieces of polyhead were found to vary from $870 \pm 30 \AA$ to $1000 \pm 30 \AA$. This indicates that the number of subunits round the polyhead was also variable. The widths of the narrowest parts of the polyhead (e.g. Pl. 2, fig. 13) were $660 \pm 30 \AA$. The average centre-centre spacing of the subunits was $72 \AA$ (23 measurements) from normal prints and 70.5 \& (30 measuremeasurements) from Pl. 3, fig. 15. The spacing of the lines of subunits down the long axis of the polyhead (lateral striations on Pl. 3, fig. 14) was $60 \AA$ (20 striations were 
$1200 \AA$ ). From Pl. 3, fig. 15, the result was also $60 \AA$. One may use this figure to calculate the centre-centre spacing of the subunits to check the direct measurements given above $\left(60 \AA\right.$ sec. $\left.30^{\circ}\right)$ the result being $69 \cdot 3 \AA$. It is considered that this figure and the value of $\mathbf{7 0 . 5} \AA$ obtained from the rotated prints (Pl. 3, fig. 15) are more accurate than the $72 \AA$ value taken from normal prints, because of the difficulty in measuring the latter properly. The average of the former two measurements is virtually $70 \AA$; this will be taken as the final value.

\section{The diameter of the tail core}

A component which might well correspond in diameter to an individual head capsomere is the tail core. This was carefully measured from micrographs of preparations of lethal mutations which produce cores with base-plates attached (Pl. 3, fig. 17). This core is far too long to fit a normal phage particle, but its diameter is $70 \AA$, which agrees with the value found for cores by other authors.

\section{The dimensions of collars and base-plates}

To assist in differentiating between collars and base-plates found detached from virions, the diameters of various star-shaped or hexagonal discs figured by other authors and in the present paper were measured. The results are given in Table 3. Objects 1-3 are hexagonal discs with apical subunits and are considered to be collars. Objects 6-8 are star-shaped and are disrupted base-plates.

Table 3. Dimensions of collars and base-plates

\section{Object}

1. Disc, Brenner \& Horne (1959), fig. 6

2. Disc, Anderson \& Stephens (1964), fig. 7-9

3. Disc, present paper, Pl. 4, fig. 23

4. Collars on intact phage, 8 measurements, Bradley, unpublished

5. Base-plates on intact phage, 8 measurements, Bradley, unpublished

6. Star, Fernandez-Moran (1962), fig. 2

7. Star, Anderson \& Stephens (1964), figs. 4, 5

8. Star, present paper, Pl. 3, fig. 20

\begin{tabular}{|c|c|c|}
\hline Max. $\AA$ & $\begin{array}{c}\text { Average } \\
\AA\end{array}$ & Min. $\AA$ \\
\hline 400 & 375 & $\mathbf{3 5 0}$ \\
\hline 4,10 & 360 & 310 \\
\hline 430 & 375 & $\mathbf{3 2 0}$ \\
\hline- & 360 & - \\
\hline - & 305 & - \\
\hline 600 & - & - \\
\hline 600 & - & 一 \\
\hline 580 & - & - \\
\hline
\end{tabular}

Note: maximum values were obtained by measuring the diameter from apex to apex and minimum values by measuring from the centres of opposite sides. On intact phages where the orientation was unknown, the value obtained was considered as an average.

\section{The base-plate and tail pins}

In the normal way, the phage base-plate and tail pins are seen in profile ( $\mathrm{Pl} .2$, fig. 10). In this micrograph one can also see two small pins pointing upwards towards the head. These are clearer in Pl. 3, fig. 18, where they are surmounted by a pyramid-like structure, which has obviously been revealed by the penetration of the negative stain into the sheath, for it is clearly an internal structure. With most negative-staining media, the base-plate and tail pins have a tendency to break up into what is usually a mass of fibres, but which on occasion takes the form of a star, shown partly bent over in Pl. 3, figs. 19, 20. It is to be noted that the tail fibres 
have remained attached to it. Anderson \& Stephens (1964) showed a particularly good micrograph of this structure; it is reproduced here in Pl. 4, fig. 21.

\section{The collar}

As with the base-plate the collar is normally seen in profile (Pl. 4, fig. 22) fitting closely to the flattened apex of the basal pyramid of the head. When detached from the virion, the collar is a six-sided disc with a morphological subunit at each apex and a central plug of protein (Pl. 4, fig. 23). A much clearer picture can be obtained by using the rotation method for printing the plate (Pl. 4, fig. 24). A careful comparison of the two micrographs is required for correct interpretation; only features which show a marked increase in contrast in the rotated print as opposed to the original, can be considered real. Thus, the hollow apical subunits are a true representation, and probably the central plug, but the ring of relatively indistinct subunits in between is an artifact. It is to be noted that there were no tail fibres attached to the collar.

Pl.4, fig. 23, is very similar to several micrographs of objects described by Anderson \& Stephens (1964) as base-plates; it will be shown below that these are, in fact, collars.

\section{DISCUSSION}

The shape of the $T 2$ phage head. The heads in Pl. 1 fig. 1 , show diagonal lines and faces in the centre portions which are clearly not hexagonal prisms. The most obvious argument against such evidence is that the heads have become distorted. However, this would be unlikely to produce straight lines, indeed a familiar pattern indicating distortion and collapse consists of an irregular band across the centre of the head (see Bradley, 1963, Pl. 1, fig. 5); also the clarity of the faces in Pl. 1, fig. 5, is not consistent with this criticism. The reason why this observation has been overlooked in the past is that most of the electron microscopy done with $\mathrm{T} 2$ type phages has involved negative staining in PTA. I showed (Bradley, 1962) that the heads collapse very easily in this medium so that clear facets will be seen only occasionally, though diagonal lines, etc., are more frequent, as mentioned above.

The present model was arrived at by trial and error and is more a working hypothesis than a definite proposal. Its accuracy may be judged by comparing the wire silhouettes with the heads; the various edges showing in the micrographs correspond closely with those on the model. Also, the angle made by the diagonal lines with the short axes of the head (Table 2), may be measured and compared with that of the model. It can be seen that such an angle would correspond to the basal angles of the isosceles triangles making up the centre portion of the head. This angle can be calculated from the appropriate average measurements (given in Table 1) or measured directly from the model (Pl. 1, fig. 4) which has been constructed according to these figures. In either case the result is $72^{\circ}$, close to the value given in Table 2.

The rotation of the two pyramids with respect to one another can, of course, produce shapes in the intermediate section which are different from that postulated. One alternative is the joining of the two other points of the rhomboids (HV and VC in Fig. 1) to form two obtuse-angled triangles bending inwards slightly. Secondly, there need not necessarily be any triangles at all, the rhomboid surfaces being warped to accommodate the curvature. These arrangements would produce more 
strain in the capsid, and since they are not consistent with the evidence of the electron micrographs, are considered to be improbable.

The average dimensions in Table 1 correlate accurately with one another. For example, the angles $\alpha$ and $\beta$ were measured independently and are not related on electron micrographs, but it can be seen from Fig. 1 that in triangle $\mathrm{DBC}, \frac{1}{2} \alpha+\beta-90^{\circ}$ should equal $90^{\circ}$. When calculated from $\alpha$ and $\beta$ in Table 1 , the result is $90 \frac{1}{2}^{\circ}$. The same degree of accuracy holds for other unrelated measurements. Indeed, if this were not so, it would not be possible to construct a model with all the dimensions correct. However, the observations in Table 1 are applicable to a bipyramidal hexagonal prism as well as to the proposed figure and cannot be used as evidence in favour of the latter.

One should note that the angles $\alpha$ and $\beta$ and the length $\mathrm{HC}$ will vary slightly according to the orientation of the pyramids. The alternatives are shown in Fig. 1 as the angles GDI and DIY and the length GI. If the model is a true one, the dimensions measured will be an average of those obtained with each orientation, since values from both pyramids were taken into account. In any case, the differences are small and outside the limit of accuracy of measurement, particularly when different negative stains are used.

This new shape will in no way change the conception of the virus as a whole. The axis to which the tail is attached still has a sixfold radial symmetry, and the head volume will be unchanged. However, one definite and important possibility must be considered, namely that the head may be pleomorphic. Cummings \& Kozloff $(1960,1962)$ claimed that the $\mathrm{T} 2$ phage can have two different head lengths according to the environment. The $15 \%$ difference is located in what was originally the prismatic portion, the two pyramids remaining unchanged. They did not postulate any change in the shape of the head, but on the basis of the present model it is easy to see how its length could be changed: any rotation of the pyramids with respect to one another could alter their distance apart. In addition, such a rotation could change the geometrical figure from a bipyramidal hexagonal prism to the proposed new one, or vice versa. Under these circumstances, therefore, there would be no reason why both head forms should not exist.

The fine structure of normal phage head and polyhead. Having re-established the shape of the head with some degree of certainty, the next step is to try and relate any capsomere structure with it. Unfortunately micrographs such as Pl. 2, figs. 8, 9 do not show a regular structure, and great care must be exercised in interpreting such detail as can be discerned, since its magnitude is near that of the background structure of the negative stain. Here the instrumental resolution is considerably better than $10 \AA$, so that the negative-stain background, which is of this order, is quite clear. This will be superimposed on any structure on the phage (magnitude about $20 \AA$ ), rendering it less clear. The best approach is to compare the background with the head structure. The first most noticeable difference is the whorled appearance shown particularly clearly in Pl. 2, fig. 9. This was visible to some extent on most heads embedded in uranyl formate, and may thus be considered real. The spacing of these concentric lines is $20 \AA$. They can only be due to capsomeres or the nucleic acid within the capsid. The latter is not as far-fetched as may at first appear since uranyl ions will penetrate the capsid and combine with the DNA, effectively staining it (Bradley, 1962). The spacing is correct and indeed one might expect the 
nucleic acid strand to be wound up in this orderly fashion. The argument against this is that the whorls are definitely beaded, suggesting $20 \AA$ capsomeres. In Pl. 2, fig. 8, possible capsomeres are more obvious, but the whorls are indistinct. It must be remembered, however, that a beaded appearance can be produced by local aggregation of a stain like a shadowing material. This would give an appearance resembling the shadowed nucleic acid strands of Kleinschmidt et al. (1962). On the whole, it is considered that the whorled lines represent the nucleic acid since capsomeres would show an ordered array which is not present in these micrographs.

The attempt at marking the individual capsomeres with antibody (Pl. 2, fig. 11) was made on the assumption that if each antibody molecule attached itself to one capsomere, their arrangement might thus be revealed. As it happened, the antibody molecules were large, and there were too few present, but it is notable that their spacing on the phage head was the same as that of the polyhead subunits.

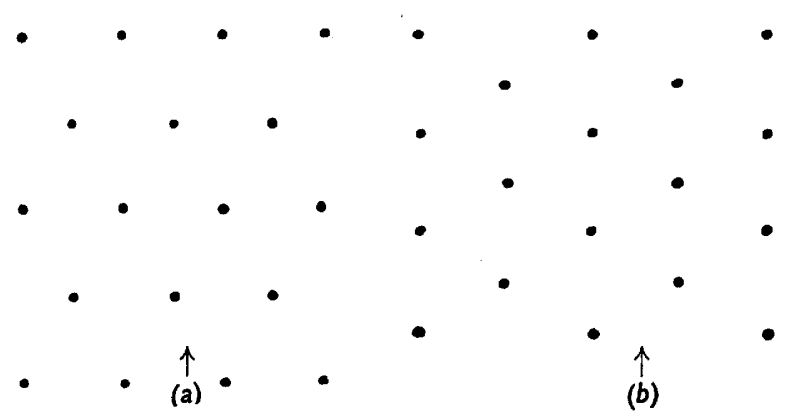

Fig. 2. Diagram of the hexagonal array of subunits in phage PVL polyhead; the arrows show the long axis of the polyhead tube; $(a)$ normal orientation, $(b)$ unusual orientation as figured by Eiserling \& Boy de la Tour (1964).

One other object must be noted: the lump of protein within the head membrane (Pl. 2, fig. 10). This is doubtless an array of molecules firmly attaching the bulky tail in a manner analogous to that of phage ZG/3A (Bradley, 1964).

At this point the structure of polyhead must be considered to see whether there is a relationship between it and the normal head structure. The array of subunits in polyhead is subject to variations in number and arrangement. The varying width of the separate pieces of polyhead (800-1000 $\AA$ ) shows that the number of subunits round the circumference is not constant. Also the orientation of the hexagonal array with respect to the long axis sometimes differs by $90^{\circ}$; the different arrangements are drawn in Fig. 2. Fig. $2 b$ is illustrated by Eiserling \& Boy de la Tour (1964) and is very unusual. Nevertheless, we are led to the inescapable conclusion that polyhead is nothing more than an assembly of head protein subunits, arranged in the most convenient packing for a cylindrical shape rather than a body with distinct facets. The most important question which arises is, why are the polyhead subunits not visible on the normal head? The most attractive answer is that the polyhead morphological subunits are composed of the same chemical subunits as the phage head proper, but that they in fact represent larger aggregates which are themselves arranged in a hexagonal packing. Such chemical units could only be 
$20 \AA$ or so in size and difficult to resolve on the phage head; they would, nevertheless, possess the same chemical and serological characteristics. On the other hand it seems almost too much of a coincidence that the diameter of the tail core and the antibody molecules in $\mathrm{Pl}$. 2, fig. 11, are both about $70 \AA$, the same size as the polyhead subunits. In short, it is impossible to postulate any definite structural relationship between polyhead and the normal phage head.

The collar and base-plate. These two components have several features in common: both are hexagonal discs, are attached to the tail core and have apical subunits. It is therefore not altogether surprising that they can easily be confused when found as isolated units. It is obviously necessary to establish distinguishing characteristics unequivocally and to ensure that objects in micrographs such as that shown in Pl. 4, fig. 23, are in fact collars.

A most valuable micrograph by Anderson \& Stephens (1964, Pl. 4, fig. 21), shows clearly that the star-shaped object has become detached from the end of the core and is thus, without question, a base-plate. This is confirmed by Pl. 3, figs. 19, 20, which show the star still attached to the base of the tail sheath. It must now be established whether the object seen in Pl. 4, fig. 23, is a collar or a base-plate in some intermediate stage of deformation, as suggested by Anderson \& Stephens (1964). Let us consider the morphological differences and their implications. First, it will be found that published electron micrographs show the star with all or most of the tail fibres attached (e.g. Fernandez-Moran, 1962) as in Pl. 4, fig. 21, whereas the suspected collars usually have no fibres at all, and very rarely one or two. Now the bond between the tail fibres and the base-plate is strong because electron micrographs show the majority of them still attached even after the base-plate has started to become disorganized. On the other hand, fibres are attached to both base-plate and collar in the intact phage only under specific conditions (see Introduction), but it is known that the bond between fibres and collar is reversible. Finally, one never finds fibres attached to the collar and not the base-plate. We may thus say that hexagonal dises with fibres attached are base-plates and objects without fibres attached are collars.

A second difference is that the star-shaped base-plates have a hole in the centre whereas the supposed collars have a plug of protein. It is clear that the hole has been caused by the base-plate having been torn from the core (as in Pl. 4, fig. 21). As with the tail fibres, the bond between the base-plate and core is strong, since these two components are usually found joined together in lysates but seldom apart. The base-plate-to-core bond only weakens under conditions of sheath contraction when the base-plate slides up the core and remains attached to the sheath; it is not released into a free state. It is thus very unlikely that base-plates will be found in a free state without a hole; this would involve snapping-off a short length of core, an improbable state of affairs. A different relationship exists between the collar and the core. The collar is attached at the weakest point on the tail, namely, where the $70 \AA$ core joins the massive tail assembly to the head. The strain here will be considerable and the core could more easily snap off than at its distal end. This would leave the collar to detach itself from the head, carrying the small plug of protein from the point where the tail penetrates the head capsid. While this argument is rather speculative it does support the contention that the hexagonal discs are collars. Apart from purely morphological considerations, particularly useful evidence is 
provided by the dimensions of the two components, both on the phage and in a free state. The results of this are summarized in Table 3 and include measurements from published micrographs taken by other authors. It can be seen that the supposed base-plates of Brenner \& Horne (1959) and Anderson \& Stephens (1964) are nearly the same size as the hexagonal disc in Pl. 4, fig. $2(420 \AA)$. The stars of Fernandez-Moran (1962), Anderson \& Stephens (1964) and Pl. 3, fig. 20 (Pl. 3, fig. 19 is clearly distorted and gives a low value) are all about $600 \AA$, much bigger than the previous value. The most pertinent comparisons are between the components in the free state and on the intact virion. The average diameter of objects 1,2 and 3 in Table 3 is about $370 \AA$, and the average for collars on intact virions is about $360 \AA$, but the average for base-plates is only $305 \AA$. Since the hexagonal discs (objects 1 , $2,3)$ show no signs of gross distortion or break-up, it seems that the discs correspond to collars rather than to base-plates, as postulated by these authors. While the differences are small, amounting to $2 \mathrm{~mm}$. on micrographs included here, unbiased measurement shows that they are real and significant.

In summary there is no doubt from the above evidence that hexagonal dises similar to Pl. 4, fig. 23, are collars. The study and comparison of many electron micrographs provides no evidence to the contrary. The collar has long been an object of some speculation, but it can now be said that its appearance is as expected, with a sixfold radial symmetry corresponding to that of the rest of the tail. A particular point of interest is the nature of the hollow apical subunits. They can, incidentally, be seen on the intact virion in $\mathrm{Pl}$. 2, fig. 10 (arrowed). It is here that the tail fibres can be released or attached by the presence or otherwise of the necessary cofactors for adsorption. Therefore this element plays an important part in the adsorption process far removed though it is from the tip of the tail. It is a remarkably thin unit being only $15 \AA$ thick, yet it is quite stable though it frequently disappears on intact virions, presumably by flattening itself against the head.

The base-plate plays a more important part in the infective process and it appears to be a rather unstable structure, readily breaking down into the star. It is not clear how this comes about or whether it is a natural process associated with cell wall penetration.

The author is grateful to Professor E. Kellenberger and his colleagues at the Institut de Biologie Moléculaire, Université de Genève, for valuable discussions and hospitality, and for providing copies of papers before publication. The author thanks Professor T. F. Anderson for permitting the publication of one of his electron micrographs, Miss M. McCulloch and Miss C. A. Dewar for technical assistance, and Professor M. M. Swann, F.R.S., for his interest and advice. The author is particularly indebted to Professor P. Wildy (Birmingham University) who suggested in a conversation some years ago that the shape of the $\mathbf{T} 2$ head was not what it appeared to be. 


\section{REFERENCES}

Anderson, T. F. (1960). On the fine structure of the temperate bacteriophages P1, P2 and P22. Proc. Eur. Reg. Conf. electron microscopy, Delft, 1960, 2, 1008.

Anderson, T. F. \& Stephens, R. (1964). Decomposition of T6 bacteriophage in alkaline solutions. Virology, 23, 113.

Bradley, D. E. (1962). A study of the negative staining process. J. gen. Microbiol. 29, 503.

Bradley, D. E. (1963). The structure of coliphages. J. gen. Microbiol. 31, 435.

Braduey, D. E. (1964). The structure of some bacteriophages associated with male strains of Escherichia coli. J. gen. Microbiol. 35, 471.

Brenner, S. \& Horne, R. W. (1959). The structure of bacteriophage. The Times Science Review, London, Autumn, 1959.

Brenner, S., Streisinger, G., Horne, R. W., Champe, S. P., Barnett, L., Benzer, S. \& REES, M. W. (1959). Structural components of bacteriophage. J. molec. Biol. 1, 281.

Cummings, D. J. \& Kozloff, L. M. (1960). Biophysical properties of bacteriophage T2. Biochem. biophys. acta, 44, 445 .

Cummings, D. J. \& Kozlofr, L. M. (1962). Various properties of the head protein of T2 bacteriophage. J. molec. biol. 5, 50.

Eiserling, F. A. \& Boy de la Tour, E. (1964). Capsomeres and other structures observed on some bacteriophages. Pathologia microbiologia (in the Press).

Epstein, R. H., Bolle, A., Steinberg, C. M., Kellenberger, E., Boy de la Tour, E., Chevalley, R., Edgar, R. S., Susman, M., Denhart, G. H. \& Lielausis, A. (1964). Physiological studies of conditional lethal mutants of bacteriophage T4D. Cold Spr. Harb. Symp. Quant. Biol. 28, 375.

Favre, R., Boy de la Tour, E., Krllenberger, E. \& Séchaud, J. (1964). Morphogenèse de la tête du bactériophage T4: observations sur les polytêtes. Proc. Eur. Reg. Conf. electron microscopy, Prague, 1964, vol. B, p. 541.

Fernandez-Moran, H. (1962). New approaches in the study of biological ultrastructure by high resolution electron microscopy. Symp. Internat. Soc. Cell Biol. (Ed. R. J. Harris), 1, 411.

Finch, J. T. (1964). The resolution of the substructure of tobacco mosaic virus in the electron microscope. J. molec. Biol. 8, 872.

KLeinschmidt, A. K., LANG, D., JACherts, D. \& ZAhn, R. K. (1962). Darstellung und Längenmessungen des gesamten desoxyribonucleinsäure-Inhaltes von T2-bakteriophagen. Biochim. biophys. acta, 62, 857.

Markham, R., Frey, S. \& Hrlus, G. J. (1963). Methods for the enhancement of image detail and accentuation of structure in electron microscopy. Virology, 20, 88.

Williams, R. C. \& Fraser, D. (1953). Morphology of seven T bacteriophages. J. Bact. 66, 458.

\section{EXPLANATION OF PLATES}

\section{Plate 1}

Fig. 1. Phage T2 in uranyl acetate with EDTA, $\times 230,000$.

Fig. 2. Phage T2 head in ammonium carbonate and sodium molybdate, $\times 200,000$.

Fig. 3. Phage T2 in uranyl nitrate; 'high-and-dry' particles in profile, $\times 200,000$.

Fig. 4. Model of proposed figure for $\mathrm{T} 2$ type heads in different orientations.

Figs. 5, 6. Phage T2 heads in uranyl acetate with EDTA with superimposed wire model, $\times 380,000$.

Fig. 7. Phage T2 head in uranyl acetate with EDTA $\times 380,000$.

\section{Plate 2}

Figs. 8, 9. Phage T4 heads in uranyl formate, $\times 400,000$.

Fig. 10. Phage $T 4$ in ammonium carbonate and sodium molybdate, $\times 300,000$. 
Fig. 11. A head of phage T4 treated with anti-T4 serum in phosphotungstate, $\times 550,000$.

Fig. 12. Polyhead (A), polysheath (B) and intact virion (C) from phage PVL in phosphotungstate, $\times 50,000$.

Fig. 13. The components in Fig. 12, $\times 333,000$.

\section{Prate 3}

Fig. 14. Polyhead from phage PVL, phosphotungstate, $\times \mathbf{8 3 3}, 000$.

Fig. 15. Marked area of Fig. 14 printed by rotation; six exposures in one revolution, $\times 333,000$.

Fig. 16. Polyhead from PVL showing hexagonal subunits (arrowed), phosphotungstate, $\times 333,000$.

Fig. 17. Core from phage $B P / 4$ in phosphotungstate, $\times 333,000$.

Fig. 18. Phage WB/5 showing detail of base-plate, phosphotungstate, $\times 333,000$.

Fig. 19. Partly disrupted base-plate of phage BP/4, phosphotungstate, $\times 833,000$.

Fig. 20. Base-plate of phage BP/4 fully disrupted to star shape, phosphotungstate, $\times 333,000$.

\section{Prate 4}

Fig. 21. Star-shaped base-plate in silicotungstate after Anderson \& Stephens (1964), with permission of Virology, Academic Press Inc., $\times$ 300,000.

Fig. 22. Phage $\mathbf{T} 4$ in potassium phosphomolybdate showing collar fitting to flattened apex of basal pyramid, $\times 333,000$.

Fig. 23. Isolated collar of phage T4 in ammonium carbonate and sodium molybdate, $\times 300,000$.

Fig. 24. Fig. 23 printed by rotation: six exposures in one revolution, $\times 450,000$. 
Journal of General Microbiology, Vol. 38, No. 3

Plate 1
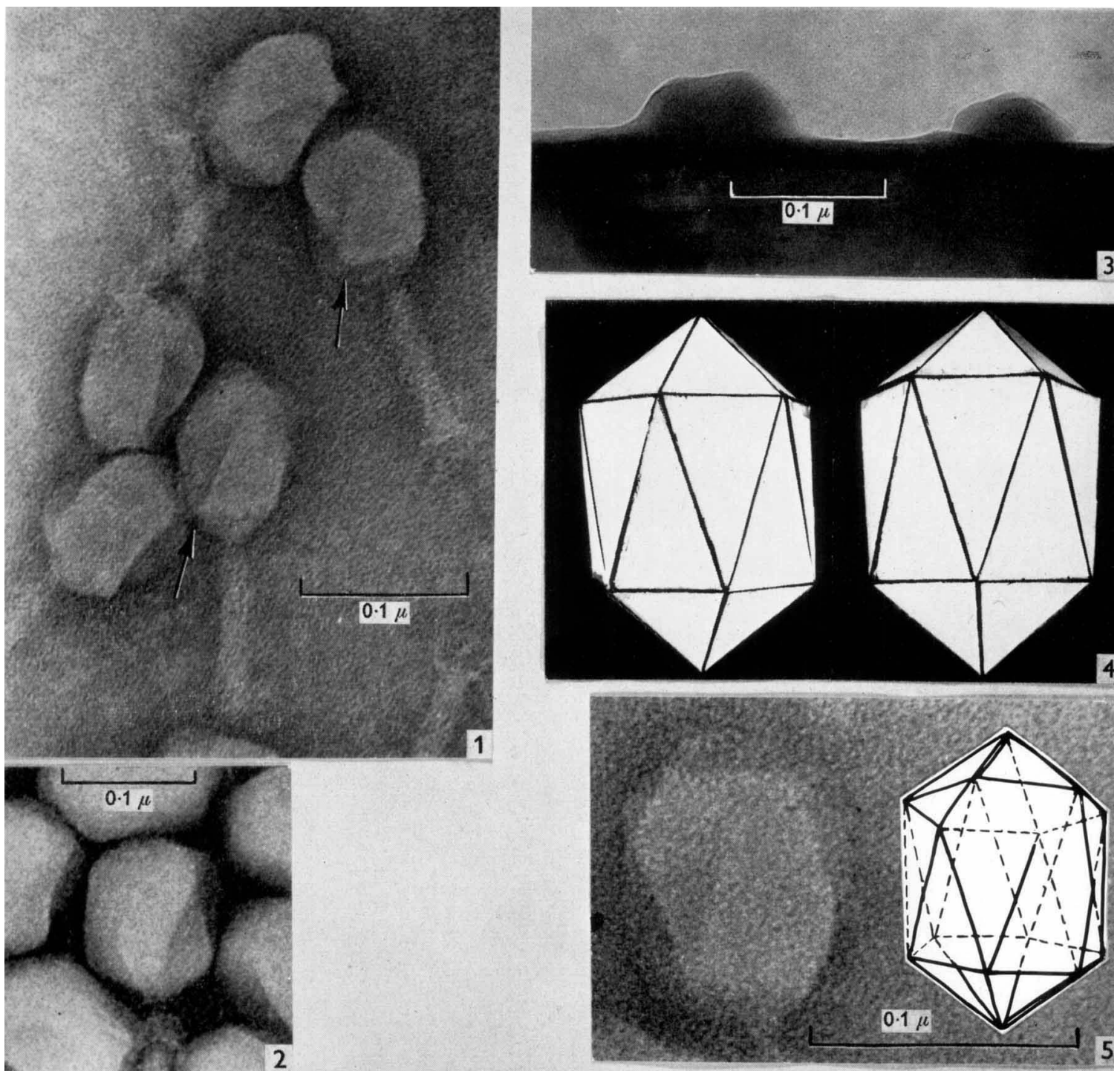

1
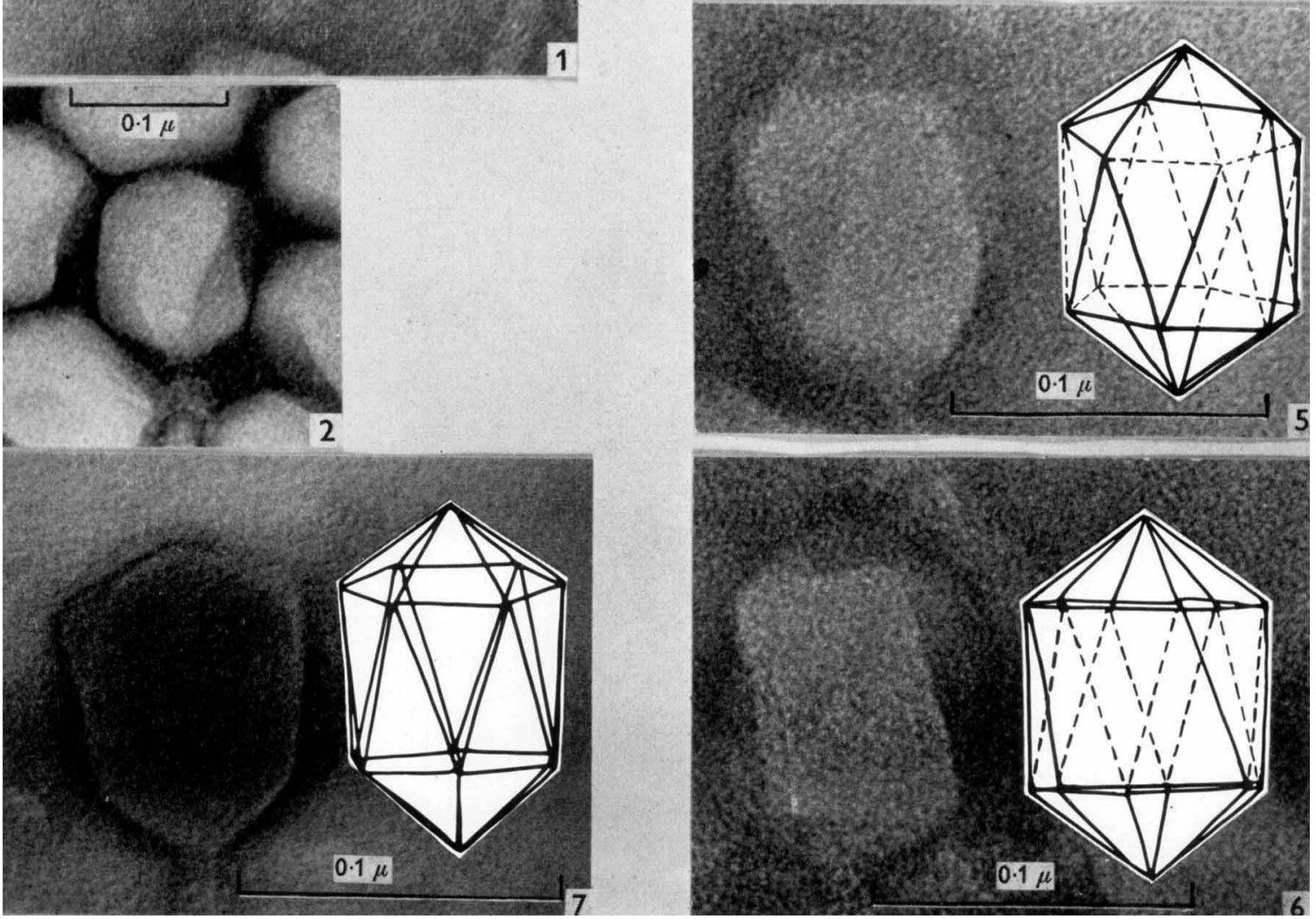
Journal of General Microbiology, Vol. 38, No. 3

Plate 2

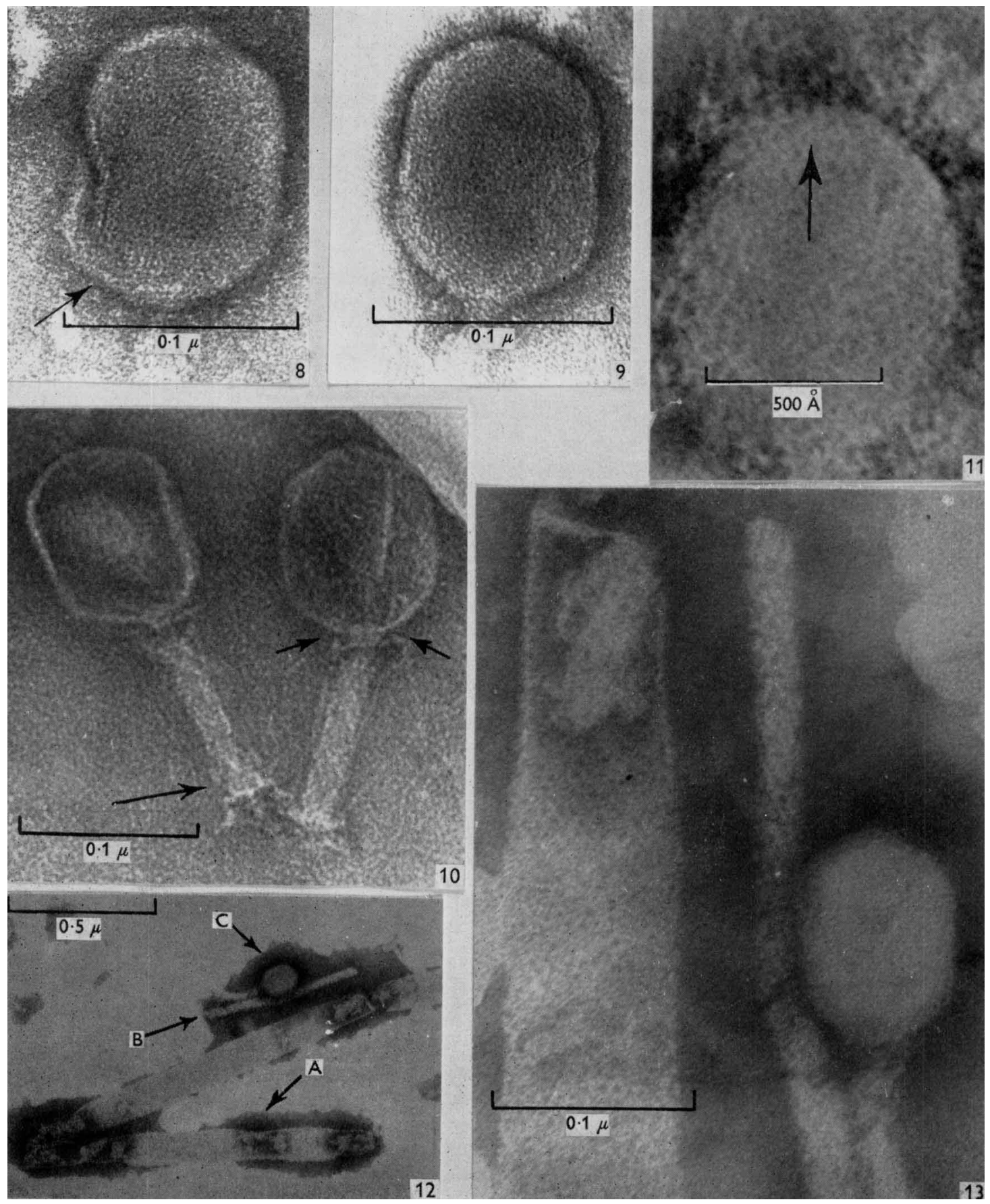

D. E. BRADLEY 
Journal of General Microbiology, Vol. 38, No. 3

Plate 3
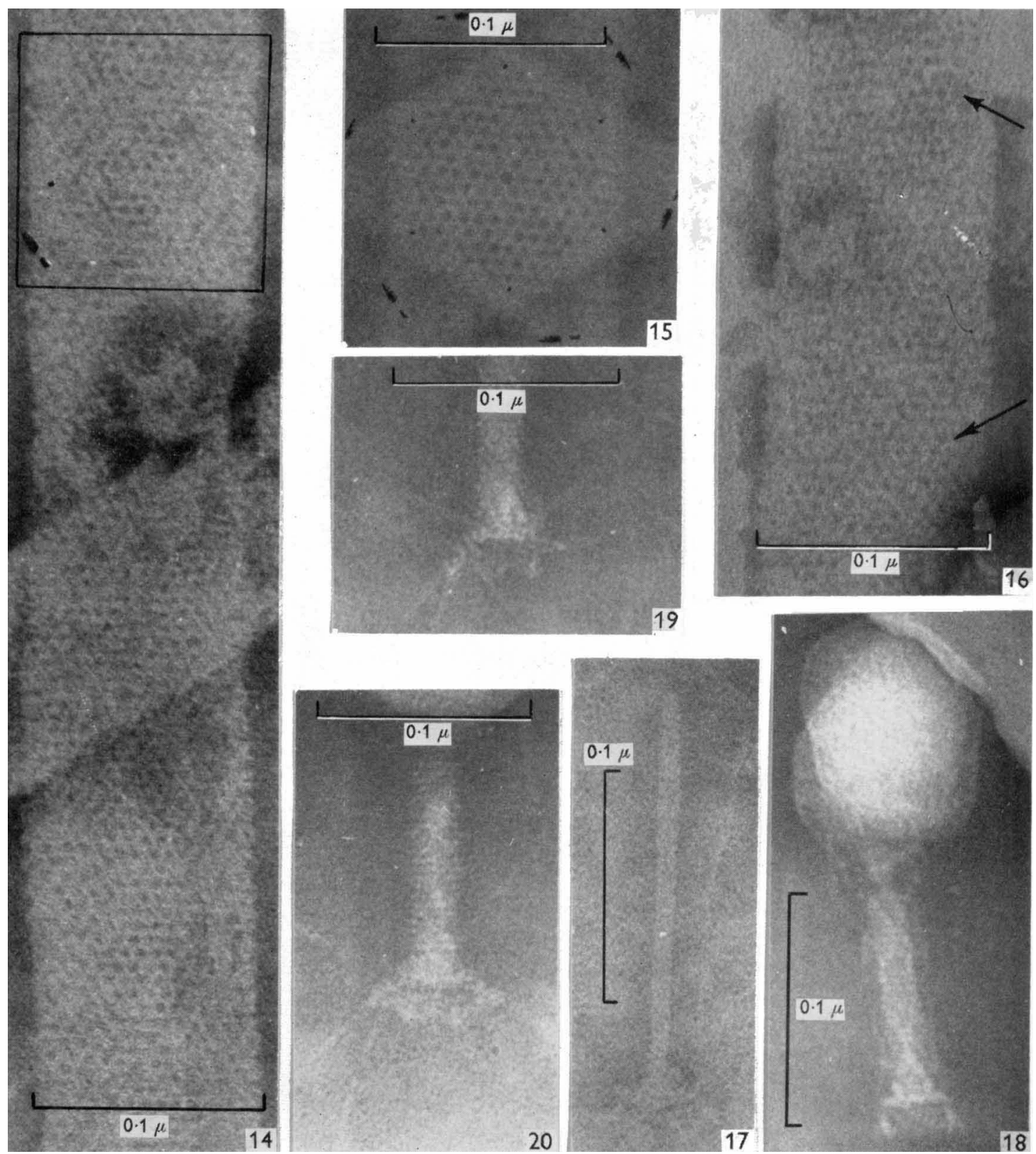

19
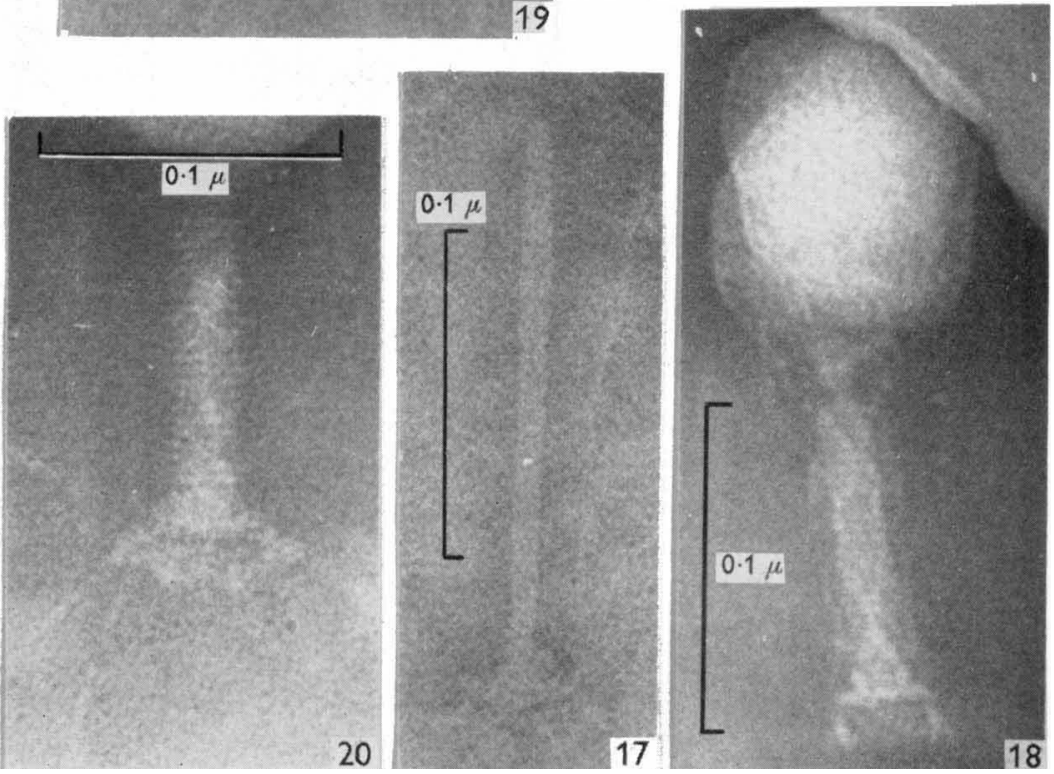

D. E. BRAILEY 


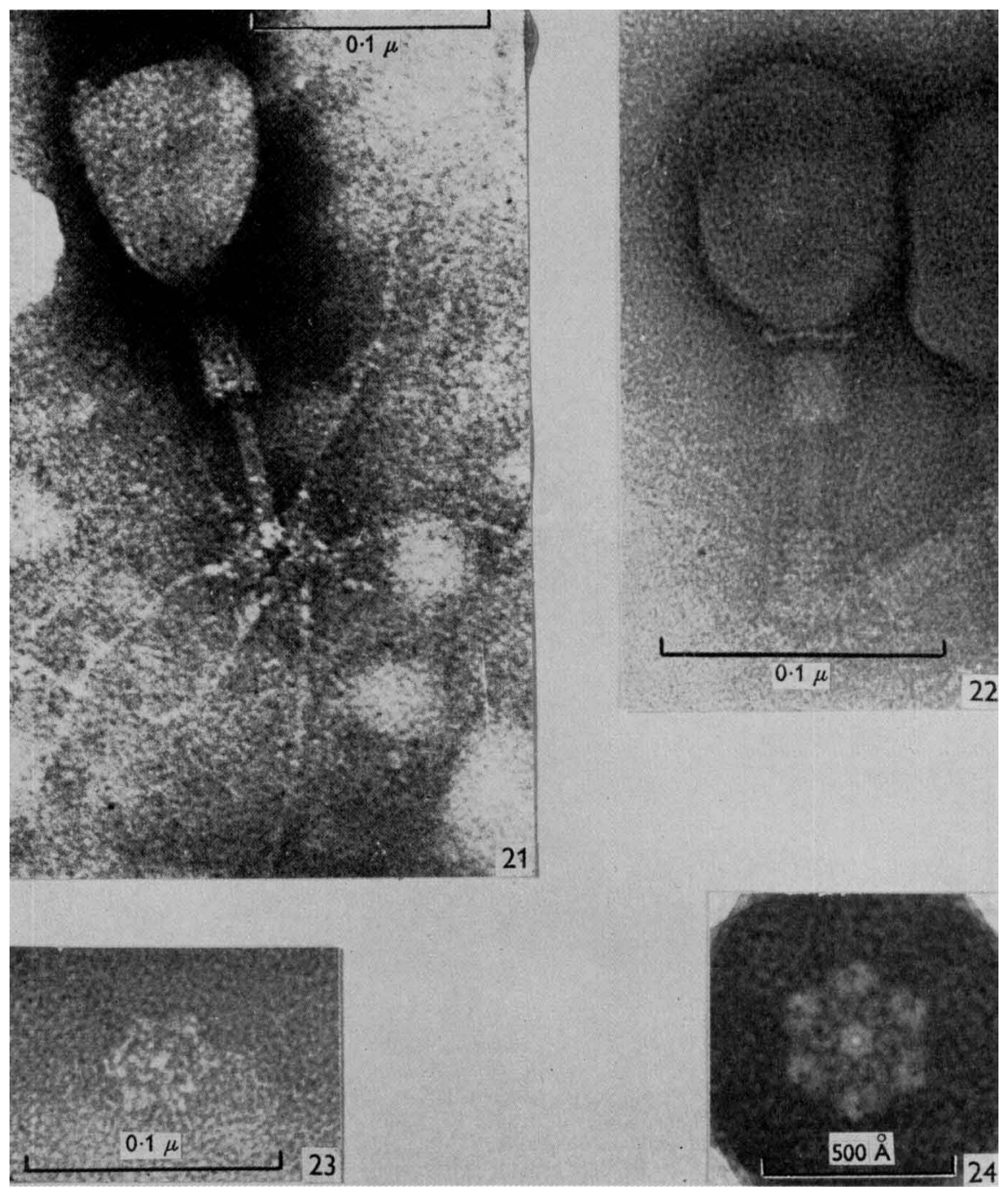

D. E. BRADLEY 